\title{
Clinical biomarker discovery by SWATH-MS based label-free quantitative proteomics: impact of criteria for identification of differentiators and data normalization method
}

Mythreyi Narasimhan ${ }^{1,2}$, Sadhana Kannan ${ }^{1}$, Aakash Chawade ${ }^{3}$, Atanu Bhattacharjee ${ }^{4}$ and Rukmini Govekar B $^{1,}$ (D)

\begin{abstract}
Background: SWATH-MS has emerged as the strategy of choice for biomarker discovery due to the proteome coverage achieved in acquisition and provision to re-interrogate the data. However, in quantitative analysis using SWATH, each sample from the comparison group is run individually in mass spectrometer and the resulting inter-run variation may influence relative quantification and identification of biomarkers. Normalization of data to diminish this variation thereby becomes an essential step in SWATH data processing. In most reported studies, data normalization methods used are those provided in instrument-based data analysis software or those used for microarray data. This study, for the first time provides an experimental evidence for selection of normalization method optimal for biomarker identification.
\end{abstract}

Methods: The efficiency of 12 normalization methods to normalize SWATH-MS data was evaluated based on statistical criteria in 'Normalyzer' — a tool which provides comparative evaluation of normalization by different methods. Further, the suitability of normalized data for biomarker discovery was assessed by evaluating the clustering efficiency of differentiators, identified from the normalized data based on p-value, fold change and both, by hierarchical clustering in Genesis software v.1.8.1.

Results: Conventional statistical criteria identified VSN-G as the optimal method for normalization of SWATH data. However, differentiators identified from VSN-G normalized data failed to segregate test and control groups. We thus assessed data normalized by eleven other methods for their ability to yield differentiators which segregate the study groups. Datasets in our study demonstrated that differentiators identified based on p-value from data normalized with Loess-R stratified the study groups optimally.

Conclusion: This is the first report of experimentally tested strategy for SWATH-MS data processing with an emphasis on identification of clinically relevant biomarkers. Normalization of SWATH-MS data by Loess-R method and identification of differentiators based on p-value were found to be optimal for biomarker discovery in this study. The study also demonstrates the need to base the choice of normalization method on the application of the data.

Keywords: Proteomic, LC-MS, SWATH, Normalization, p-value, Fold change

\footnotetext{
*Correspondence: rgovekar@actrec.gov.in

${ }^{1}$ Advanced Centre for Treatment, Research and Education in Cancer, Tata

Memorial Centre, Kharghar, Navi Mumbai 410210, India

Full list of author information is available at the end of the article
} 


\section{Background}

Liquid chromatography-mass spectrometry (LC-MS) based quantitative proteomic profiling has substantially contributed to identification of disease biomarkers for improved diagnosis/better prognostication or to monitor response to therapy [1-3]. This is achieved through assessment of the ability of differentiators, identified by quantitative proteomics, to segregate the comparison groups distinctly by cluster analysis-an essential feature of biomarkers. Success of this process depends not only on selection of appropriate clinical samples and sample processing strategies, but also on mass spectrometry based-factors such as the depth of LC-MS profile and occurrence of instrumental or non-instrumental errors in the data. Therefore, use of mass spectrometers with capabilities for in-depth profiling and data processing strategies which reduce biases, errors and optimize the desired outcome is necessary.

The recent feature in MS-Sequential window acquisition of all theoretical fragment-ion spectra (SWATH) provides in depth profiling by data independent acquisition (DIA) [4]. It is preferred for profiling clinical samples, as in data or information-dependent acquisition (IDA) data from low expressers is lost permanently [5]. SWATH not only provides for fragmentation of almost all ions but also for re-interrogation of data, after detection capabilities are improvised to identify more number of proteins [4]. These features are conducive to profiling of clinical samples which are available in amounts insufficient for enrichment and are unavailable for reanalysis. A testimony to this is the wide use of SWATH-MS in clinical proteomics after its discovery in 2012. PubMed results show that $44 \%(20 / 45)$ of the SWATH-MS studies on clinical samples published till date are aimed at biomarker discovery or therapeutic target identification.

However, a feature in quantification by SWATH-MS, if overlooked, can hinder biomarker identification. Unlike labelled quantification by IDA wherein all samples for relative quantification are run together, in label-free quantification by SWATH, each sample from the comparison group is run individually in MS. This increases the probability of both systematic and random error. Intervention to reduce these variations by 'normalization' is thus a prerequisite to subsequent analysis of SWATH data for identification of differentiators. The data from reported SWATH-MS studies is normalized using either methods provided by the MS instrument-based software or those used to normalize microarray data [6-9]. As the source of systematic bias differs between MS and microarray, it is essential to experimentally validate the appropriate normalization strategy for SWATH data.

The present study was undertaken to experimentally identify an appropriate normalization method for SWATH-MS data. The statistical tool 'Normalyzer', which compares the efficiency of diverse methods to normalize 'omics' data based on statistical criteria [10], was used to achieve the same. Fu et al. [11] in their study to identify the optimal analysis chain have reported total ion current normalization as optimal for SWATH-MS data based on statistical end-point. Further, considering the wide application of SWATH-MS in biomarker identification, in this study we have supplemented the statistical evaluation with biologically relevant criteria of precise stratification of comparison groups by cluster analysis. Towards this (a) Normalization of data was assessed using 'Normalyzer' to identify the optimal method of normalization based on statistical criteria (b) from the data normalized by different methods in Normalyzer, differentiators between comparison groups were identified based on p-value, fold change and combination of both. The potential of these differentiators to segregate comparison groups distinctly, was assessed by cluster analysis. Detection of optimal method for normalization of SWATH-MS data and optimum criteria for identification of differentiators would have an impact on biomarker discovery.

\section{Methods}

The scheme of experiments employed to identify the normalization strategy optimum for SWATH-MS data is depicted in Fig. 1. It involves:

A. Inclusion of a quantitatively defined dataset from public domain, generated from hybrid of peptides from three different sources mixed in defined proportions, to serve as a 'reference set'. Generation of datasets using K562 cells for quantitation by SWATH-MS, referred to as 'study set' which includes one set with smaller number of samples and two sets with larger number of samples. Further, inclusion of two datasets from public domain comprising of larger sample size, to serve as 'validation set' to confirm the findings in the study set.

B. SWATH-MS analysis of reference, study and validation set.

C. Normalization of SWATH-MS data obtained from reference, study and validation sets using methods in Marker view software and Normalyzer and identification of optimum method of normalization based on statistical criteria.

D. Identification of differentiators from this normalized data based on criteria of $p$ value, fold-change and both, followed by cluster analysis of these differentiators. 


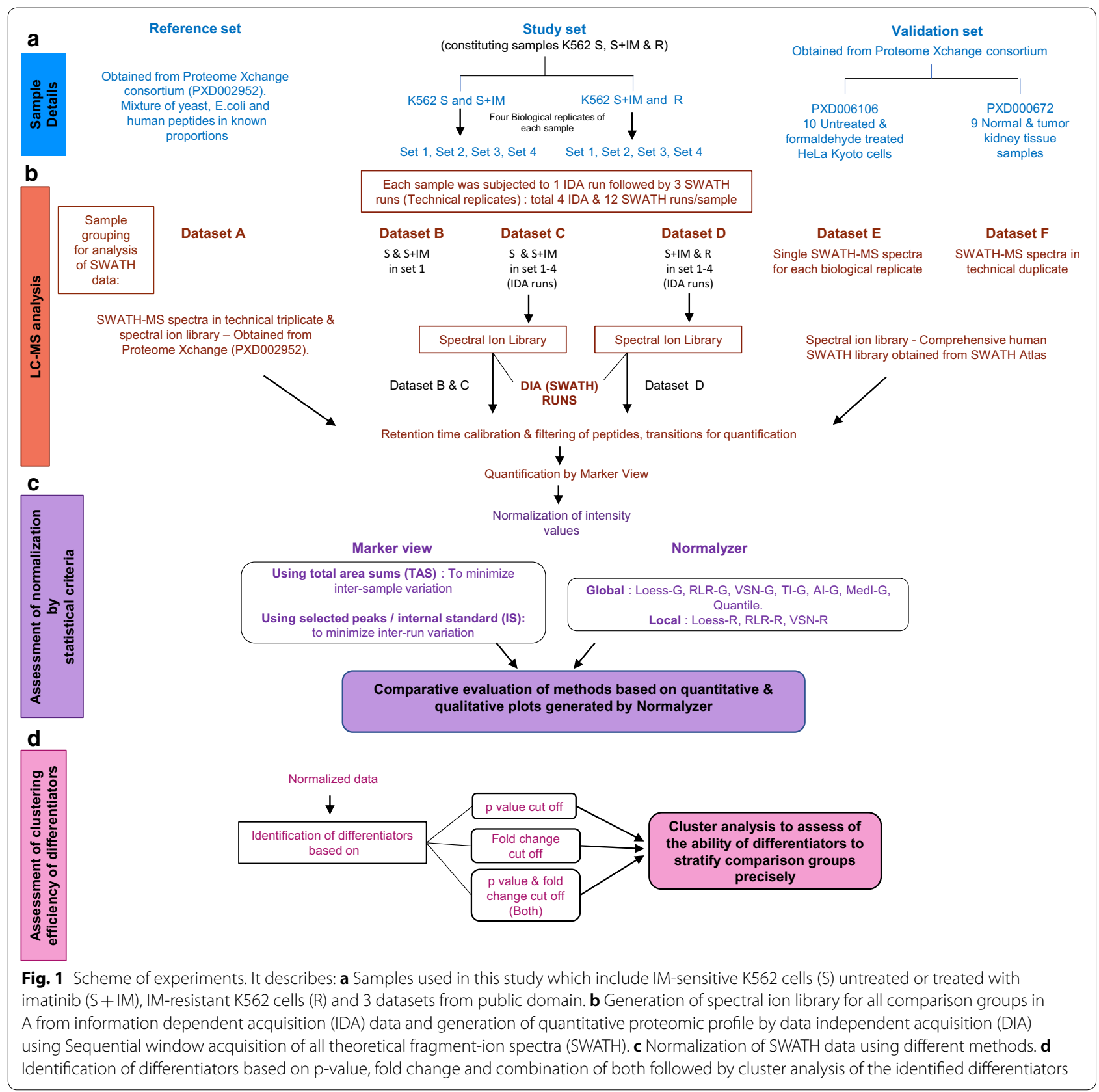

\section{Details of samples for SWATH-MS analysis}

Details of samples used for SWATH-MS analysis are summarized in Fig. 1a. The reference set was obtained from data published by Navarro et al. [12] wherein samples were prepared by mixing known proportions of constituent proteome (i.e. with known fold-difference in quantities). Samples with a hybrid of human, yeast and E. coli peptides referred to as HYE124 had differences in relative proportions of the constituent peptides and served as control $(65 \% \mathrm{w} / \mathrm{w}$ human, $30 \% \mathrm{w} / \mathrm{w}$ yeast, $5 \%$ $\mathrm{w} / \mathrm{w}$ E. coli peptides) and test $(65 \% \mathrm{w} / \mathrm{w}$ human, $15 \% \mathrm{w} / \mathrm{w}$ yeast, $20 \% \mathrm{w} / \mathrm{w}$ E. coli peptides). SWATH runs of these samples in technical triplicate and their corresponding spectral ion library deposited in Proteome Xchange consortium (identifier-PXD002952), was used for SWATH data analysis.

A 'study set' was generated in our laboratory using K562, an erythroleukemic cell line (generous gift from Dr. Tadashi Nagai, Jichi Medical University, Tochigi, Japan). It was maintained in RPMI 1640 medium supplemented with $10 \%$ FBS and $1 \%$ antibiotic (Gibco, Thermo Fisher Scientific, USA). K562 harbours BCR/ABL 
oncogene which encodes a constitutively active tyrosine kinase, whose activity is inhibited by the small molecular inhibitor imatinib (IM). Inhibition of BCR/ABL activity by imatinib is known to cause quantitative changes in the proteome of $\mathrm{K} 562$ cells $[13,14]$.

Thus IM-sensitive K562 cells (S) untreated or treated with imatinib $(\mathrm{S}+\mathrm{IM})$ and IM-resistant K562 cells (R) were analyzed. For $\mathrm{S}$ cells, treatment with imatinib was carried out at $0.75 \mu \mathrm{M}$ concentration for $12 \mathrm{~h}$, a condition observed to inhibit BCR/ABL activity without compromising on cell viability (data not shown). $\mathrm{R}$ cells were always maintained in medium containing $0.75 \mu \mathrm{M}$ imatinib. SWATH-MS profiles were generated for four biological replicates of S, S + IM and R, each run-in triplicate (Fig. 1a, b).

The 'validation set' constituted SWATH data deposited by Tan et al. [15]. and Guo et al. [16] in Proteome Xchange consortium with identifiers PXD006106 and PXD000672, respectively. SWATH runs of ten biological replicates of HeLa Kyoto cells untreated (UT) and treated with formaldehyde (FA) were obtained from PXD006106 while duplicate SWATH runs of normal (N) and tumorous ( $\mathrm{T}$ ) kidney tissue samples from nine patients were obtained from PXD000672.

\section{Preparation of K562 lysates for LC-MS analysis}

To prepare whole cell lysate, $1 \times 10^{6}$ cells were suspended in $100 \mu \mathrm{l}$ SDS buffer (10\% glycerol, $2 \%$ SDS, $5 \%$ $\beta$-mercaptoethanol and $62.5 \mathrm{mM}$ tris $\mathrm{pH} 6.8$ ), boiled for $10 \mathrm{~min}$ and centrifuged at $13,000 \times g$ for $15 \mathrm{~min}$. The supernatant was collected and acetone precipitated with $1 \mathrm{ml}$ chilled acetone to remove detergents. Protein pellet thus obtained was denatured by resuspending in $6 \mathrm{M}$ urea and protein concentration was determined by Bradford assay [17]. $10 \mu \mathrm{g}$ protein was subjected to in-solution trypsin digestion. Briefly, the denatured proteins were reduced by incubating with $200 \mathrm{mM}$ dithiothreitol (DTT) for $1 \mathrm{~h}$ at room temperature. It was followed by alkylation with $200 \mathrm{mM}$ iodoacetamide (IAA) for $1 \mathrm{~h}$ in dark. Before trypsin digestion, urea concentration was adjusted to $0.6 \mathrm{M}$ using $1 \mathrm{mM} \mathrm{CaCl}$. In-solution digestion was carried out by adding proteomic grade trypsin (Sigma Aldrich, USA) in the ratio of 1:50 trypsin: protein $(\mathrm{w} / \mathrm{w})$ and incubated for $16 \mathrm{~h}$ at $37^{\circ} \mathrm{C}$. Peptides were then desalted using C18 spin columns (Pierce, Thermo Fisher Scientific, USA), dried in a speed vac and reconstituted with $0.1 \%$ Formic acid (FA) in water to get a final concentration of $0.5 \mu \mathrm{g} / \mu \mathrm{l}$.

\section{LC-MS/MS data acquisition for the study set}

Each sample in the study set was spiked with $1 \mathrm{pmol} / \mu \mathrm{l}$ of digested Escherichia coli $\beta$-galactosidase $(\beta$-gal) peptides (Sciex, USA), before injection, which served as internal standard. The samples were then injected into Eksigent ekspertTM nano-LC 400 with cHiPLC $^{\circledR}$ system, with trap column $(200 \mu \mathrm{mX} 0.5 \mathrm{~mm})$ and analytical column $(75 \mu \mathrm{mX} 15 \mathrm{~cm})$, both packed with $3 \mu \mathrm{l}$ ChromXp C18 $(120 \AA)$. For reverse phase HPLC, $0.1 \%$ FA in water and $0.1 \%$ FA in acetonitrile (ACN) served as solvent $A$ and $B$ respectively. A gradient elution of $225 \mathrm{~min}$, with increasing percentage of mobile phase $B$ was used to elute the peptides at a flow rate of $300 \mathrm{nl} / \mathrm{min}$. Eluate from the column was analyzed in a positive ion mode on Triple TOF $5600+($ Sciex, USA) mass spectrometer.

Each sample was subjected to 1 IDA run for spectral ion library generation followed by 3 DIA (SWATH) runs, which served as technical replicates. Thus, with four biological replicates, K562 S, S + IM and R cells had 4 IDA runs and 12 SWATH runs each (Fig. 1b). IDA mode involved a survey scan over a mass range of 350$1250 \mathrm{~m} / \mathrm{z}$ and MS/MS scan over $200-1800 \mathrm{~m} / \mathrm{z}$ for top 30 precursor ions with rolling collision energy, $50 \mathrm{mDa}$ mass tolerance and accumulation time of $250 \mathrm{~ms}$ for MS and about $50 \mathrm{~ms}$ for MS/MS.

For DIA-SWATH acquisition, the instrument was tuned to a looped product ion mode. A sequential isolation window width of $25 \mathrm{~m} / \mathrm{z}$ (with $1 \mathrm{~m} / \mathrm{z}$ overlap) covering a mass range of $350-1250 \mathrm{~m} / \mathrm{z}$ was set, resulting in 36 overlapping windows. The accumulation time was $50 \mathrm{~ms}$ for MS scan and $80 \mathrm{~ms}$ for MS/MS scan, thereby making a total cycle time of about $3 \mathrm{~s}$. The conditions used to generate data by Navarro et al. [12]. Guo et al. [16] and that used to generate data experimentally in this study were comparable, while data generated by Tan et al. [15] used 64 variable wide precursor ion selection window. Further, samples in the reference set and validation set were spiked with indexed retention time (iRT) peptides for retention time calibration while those in the study set were spiked with $E$. coli $\beta$-gal peptides.

\section{Generation of spectral ion library for the study set}

The reference set from Navarro et al. [12] was referred to as Dataset A. The data acquired from S, S + IM and R sets were further grouped for comparison into datasets (Fig. $1 \mathrm{~b}$ and Table 1). Only one out of the four sets of $S$ and $\mathrm{S}+\mathrm{IM}$ each, was considered as dataset $\mathrm{B}$ while all four together as dataset C. All four sets of $S+I M$ and $R$ were included in dataset $\mathrm{D}$. The validation sets from Tan et al. [15] and Guo et al. [16] were referred to as dataset E and $F$ respectively.

A common spectral ion library was generated for datasets $B$ and $C$ while a separate library was created for dataset D. The spectral ion library for datasets $B, C$ and $\mathrm{D}$ was generated by pooling the IDA runs of the corresponding biological replicates and analysing in Protein Pilot software v4.5 (Sciex, USA) with paragon algorithm, 
Table 1 Details of datasets

\begin{tabular}{|c|c|c|c|}
\hline Datasets & Source & Constituents & Purpose-in this study \\
\hline Dataset A & Pride ID_PXD002952 & $\begin{array}{l}3 \text { samples of } 65 \% \text { human, 30\% yeast, } 5 \% \text { E. } \\
\text { coli peptides (Control) } \\
3 \text { samples of } 65 \% \text { human, 15\% yeast, } 20 \% \text { E. } \\
\text { coli peptides (Test) }\end{array}$ & $\begin{array}{l}\text { Reference set-a well-defined dataset with } \\
\text { predictable quantification }\end{array}$ \\
\hline Dataset B & In vitro experiments carried out in this study & $\begin{array}{l}3 \text { samples of K562/S cells (Control) } \\
3 \text { samples K562/S + IM cells (Test) }\end{array}$ & $\begin{array}{l}\text { Study set to check comparability of observa- } \\
\text { tions in defined (A) versus undefined (B) } \\
\text { datasets }\end{array}$ \\
\hline Dataset C & In vitro experiments carried out in this study & $\begin{array}{l}12 \text { samples of K562/S cells (Control) } \\
12 \text { samples K562/S + IM cells (Test) }\end{array}$ & $\begin{array}{l}\text { Larger dataset }(C) \text { to check the application of } \\
\text { observations from small dataset }(B)\end{array}$ \\
\hline Dataset D & In vitro experiments carried out in this study & $\begin{array}{l}12 \text { samples of K562/S + IM cells (Control) } \\
12 \text { samples K562/R cells (Test) }\end{array}$ & $\begin{array}{l}\text { Larger dataset (D) to check the consistency of } \\
\text { observations in independent large datasets }\end{array}$ \\
\hline Dataset E & Pride ID_PXD006106 & $\begin{array}{l}10 \text { samples of untreated HeLa Kyoto cells } \\
\text { (Control) } \\
10 \text { samples of formaldehyde treated HeLa } \\
\text { Kyoto cells (Test) }\end{array}$ & $\begin{array}{l}\text { Validation set to check the consistency of } \\
\text { observations in independent large datasets }\end{array}$ \\
\hline Dataset F & Pride ID_PXD000672 & $\begin{array}{l}18 \text { non-tumorous kidney tissue samples } \\
\text { (Control) } \\
18 \text { tumorous kidney tissue samples (Test) }\end{array}$ & $\begin{array}{l}\text { Validation set to check the consistency of } \\
\text { observations in independent large datasets }\end{array}$ \\
\hline
\end{tabular}

to obtain protein identities. The parameters used were as follows: Cysteine alkylation-IAA, digestion-trypsin and no special factor was chosen. The search effort was set to 'thorough ID' and false discovery rate (FDR) analysis was enabled. Proteins identified with 1\% FDR were considered. The search was carried out against UniProt database (November 2016 release) containing human proteins as well as $E$. coli $\beta$-gal. The result (group) file thus generated served as the spectral ion library. For dataset A the spectral ion library deposited by Navarro et al. [12], generated by pooling individual libraries of constituent human, yeast and $E$. coli peptides, was used. For datasets $\mathrm{E}$ and $\mathrm{F}$ comprehensive human SWATH library with about 10,000 proteins deposited in SWATH Atlas by Rosenberger et al. [18] was used.

\section{SWATH data analysis}

Spectral alignment and targeted data extraction of the swath runs of all six datasets were carried out in Peak View 2.2 software using MS/MS ALL with SWATH acquisition microapp (Sciex, USA). Proteins from spectral ion library identified with $1 \%$ FDR were first imported into Peak View 2.2 software. Retention time calibration was carried out using iRT peptides for datasets A, E and $\mathrm{F}$ and $E$. coli $\beta$ - gal peptides for datasets B-D. Processing settings were used to filter the ion library, where up to 6 peptides per protein and 6 transitions per peptide with peptide confidence threshold of $99 \%$ and FDR of $1 \%$, were chosen for quantification. Modified peptides were excluded from extraction. Extracted ion chromatogram (XIC) window was set to $5 \mathrm{~min}$ for datasets $\mathrm{A}, \mathrm{B}, \mathrm{C}, \mathrm{E}$, $\mathrm{F}$ and $15 \mathrm{~min}$ for dataset D with XIC width of $50 \mathrm{ppm}$. The MS/MS extracted peak areas from the filtered results were exported to Marker View software v1.3 (Sciex, USA) for quantification. The marker view output raw data file with list of proteins and their peak areas were used for further analysis.

\section{Normalization of SWATH Data}

The raw data of all datasets was processed and analyzed in Normalyzer (Fig. 1C), wherein it was log2 transformed and then normalized globally (G) or locally (R) using 10 statistical methods. Global normalization is carried out without consideration of affiliation of the sample such as replicate, control group, test group, etc. [10]. In SWATH-MS since each sample is run individually, errors can arise irrespective of their origin. Thus, in the present study global normalization methods were included. However, since the study focuses on identification of normalization method conducive to biomarker identification, retention of distinguishing features of the comparison groups was necessary while normalizing the data. This was achieved by including local normalization methods for analysis [10]. The normalization methods include locally estimated scatterplot smoothing (Loess-R, Loess-G) which assumes non-linear relationship between the bias in the data and magnitude of protein intensity; robust linear regression (RLR-R, RLR-G) which assumes that the bias in data is linearly dependent of the magnitude of the measured protein intensity; variance stabilization normalization (VSN-R, VSN-G) which aims at making the sample variances nondependent from their mean intensities and bringing the samples onto the same scale; quantile normalization which forces the distribution of the samples to be the same; total intensity (TI-G), average 
intensity (AI-G) and median intensity (MedI-G) normalization methods wherein intensity of each variable is divided by sum of intensities, mean of sum of intensities, median intensities of all variables respectively $[8$, 10].

Marker view v1.3 along with quantitation also provides options for sample normalization using either total area sums (TAS) wherein total area of all peaks in a sample is considered or using area of the selected peaks or internal standard (IS). In this study spiked iRT peptides and trypsin digest of E. coli $\beta$ - gal served as an internal standard for dataset $\mathrm{A}, \mathrm{E}, \mathrm{F}$ and datasets $\mathrm{B}-\mathrm{D}$ respectively. In TAS as well as IS normalization, the peak areas of each sample were normalized by multiplying with its corresponding scale factor. The scale factor for TAS method was obtained by dividing the average of total area of all samples by the total area of each sample while for IS method the average area of internal standard of all samples was divided by the area of internal standard of each sample. Data normalized by the above two methods i.e. TAS and IS was log2 transformed before running through Normalyzer, to generate the evaluation report.

The normalization efficiency of all 12 methods was assessed through 'Normalyzer' quantitatively by pooled intragroup coefficient of variation (PCV) and qualitatively by relative log expression (RLE) plot as reported in earlier studies $[8,10]$.

\section{Identification of differentiators from normalized data and cluster analysis}

Differentiators were identified from the data of all datasets normalized by 12 methods based on the criteria of p-value, fold-change and a combination of both (Fig. 1d). To obtain p-value, $\log 2$ transformed data, normalized by different normalization methods from comparison groups were assessed by Student's t-test using IBM SPSS statistics 21. Differences in protein intensities with p-value $\leq 0.05$ were considered statistically significant and chosen as differentiators. The fold change difference in protein levels was calculated from the peak area values and a cut-off of 1.5 -fold change was applied. Further, the efficiency of differentiators obtained from data normalized using the 12 methods to segregate the comparison groups was assessed by cluster analysis. The peak areas of differentiators identified using p-value $(\leq 0.05)$, fold change ( 1.5 fold) and combination of both were used as inputs for cluster analysis (Fig. 1d) in Genesis software v.1.8.1. Hierarchical clustering was performed with the following parameters: Agglomeration rule-Average linkage WPGMA and Calculation parameters - Cluster experiments.

\section{Results}

Identification and quantitation of proteins by SWATH-MS In this study, each of the four biological replicates of K562 S, S + IM and R, underwent one IDA run for the generation of spectral ion library followed by three DIA runs for SWATH-MS analysis, thereby resulting in a total of 4 IDA and 12 DIA runs for K562 S, S + IM and $\mathrm{R}$ each. Samples with improper chromatogram were eliminated from analysis sets leaving 11 runs each in $S$ and $R$ in datasets $C$ and $D$ respectively (Fig. $1 b$ ). In dataset F, there were 2 technical replicates for each sample. Upon spectral alignment and filtering of ion library, 4404, 1450, 1757, 1808, 7057 and 5316 proteins that fulfilled the criteria (described in Methods under 'SWATH data analysis') were further used for quantification of datasets A, B, C, D, E and F respectively. Quantities of the identified proteins were further assessed for variation.

\section{Assessment of variation in un-normalized data}

The quantified $\log 2$ transformed 'un-normalized' data of each dataset was evaluated based on RLE plot, which assesses the inter- and intra-group alignment of the replicates qualitatively. In RLE plot, samples should be aligned around zero. Any deviation would indicate discrepancies in the data [10]. Among the datasets constituted of single set of samples, alignment around zero was seen in all the representative samples of dataset A (Fig. 2a) and $50 \%$ of those in dataset B (Fig. 2b). Datasets C (Fig. 2c), D (Fig. 2d) and F (Fig. 2f) comprising of multiple sets, showed considerable deviation from zero in replicates as well as between groups in RLE plots, indicating the need for normalization of SWATH-MS data.

\section{Identification of optimum method for normalization using 'Normalyzer'}

The efficiency of 12 different normalization methods to normalize datasets $\mathrm{A}-\mathrm{F}$, was assessed quantitatively and qualitatively in 'Normalyzer' using PCV and RLE plots respectively. PCV reflects the ability of a normalization method to decrease intragroup variation between technical and/or biological replicates [8]. The results indicated that, VSN-G-normalized data consistently showed lesser intra-group variation in all datasets compared to data normalized by other methods (Fig. 3I). Additionally, in datasets B-F VSN-R normalized data also reduced intra group variation. Further, qualitative assessment of the normalization methods with lowest PCV (VSN-G and VSN-R) by RLE plot indicated that only VSN-G showed good inter and intra group alignment among the replicates in all datasets (Fig. 3II). Thus, VSN-G was identified 

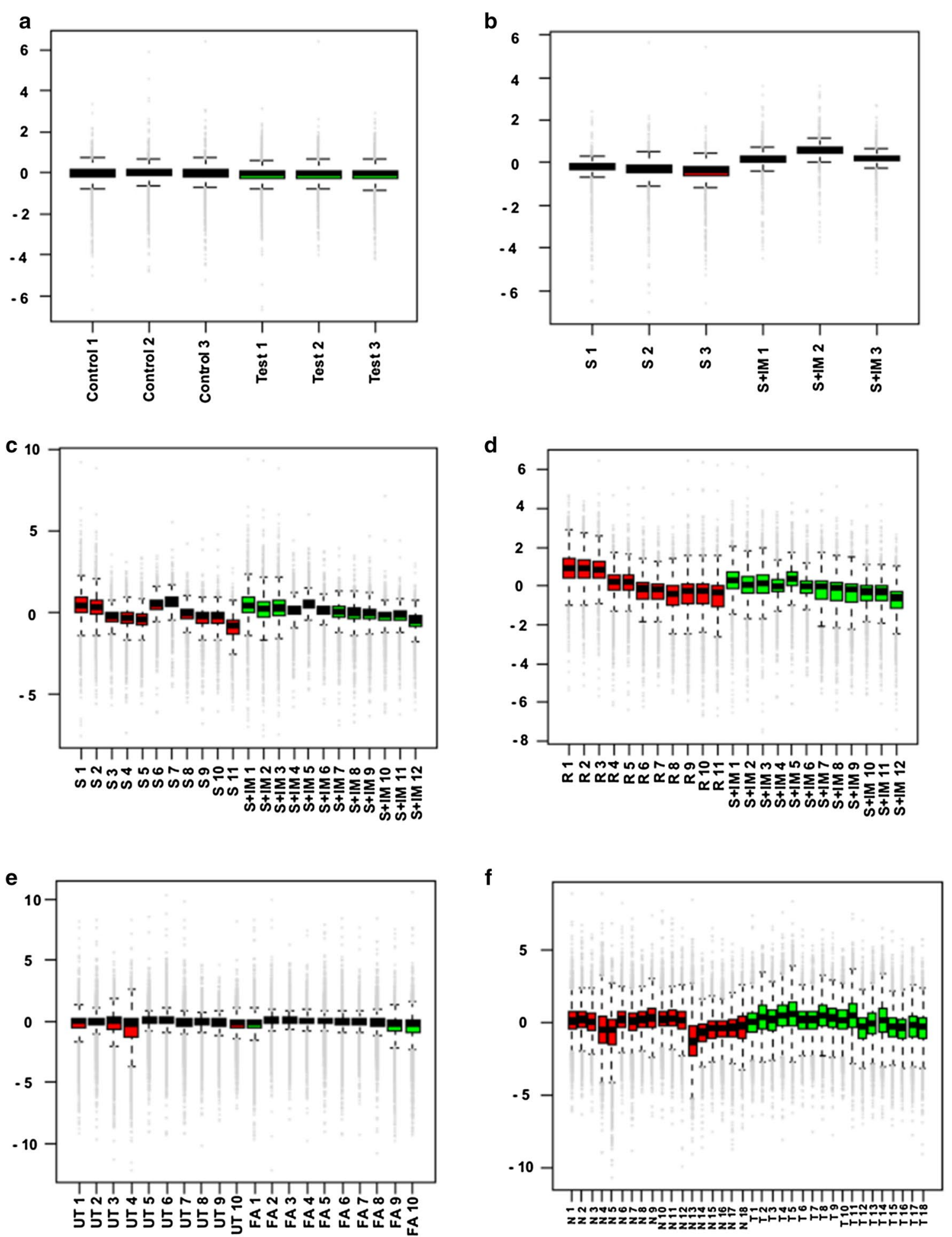

Fig. 2 Analysis of unnormalized SWATH data for datasets A-F (a-f) by RLE plot: Qualitative assessment of the spread of data shows that the test and control groups vary in their spread of values in all datasets except $\mathbf{a}$ and $\mathbf{e}$ 


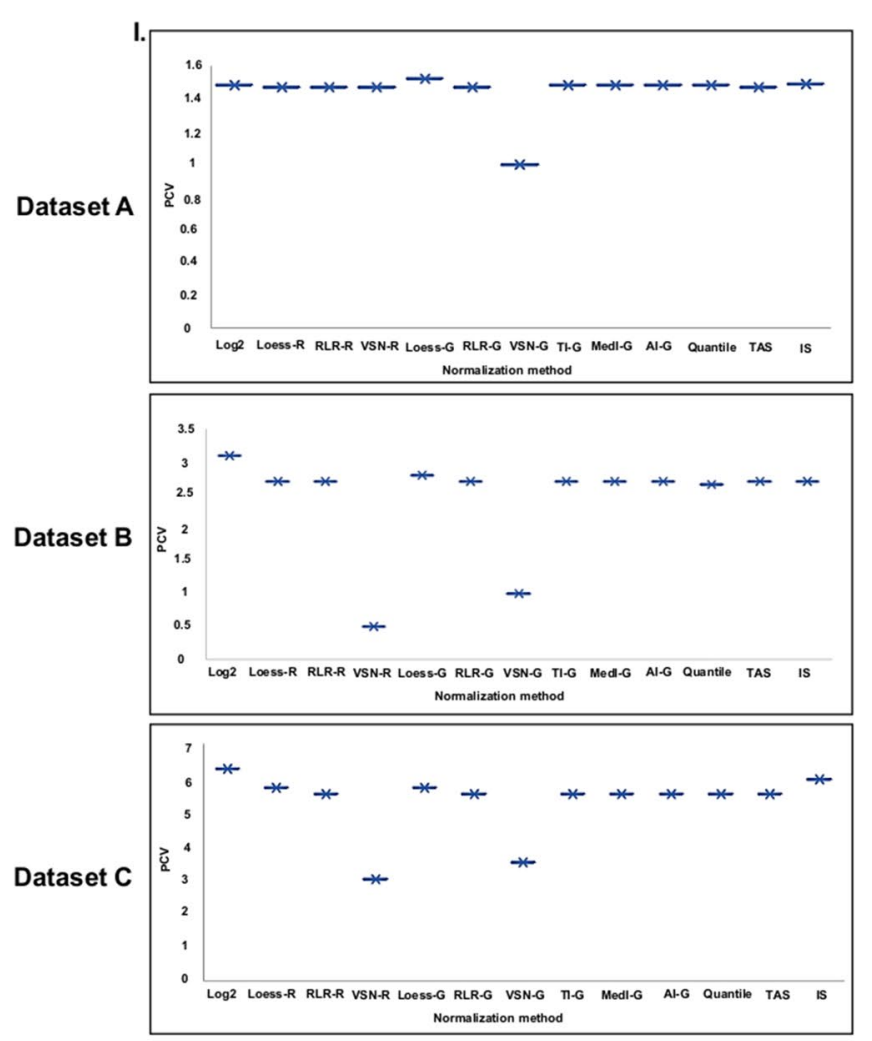

II.
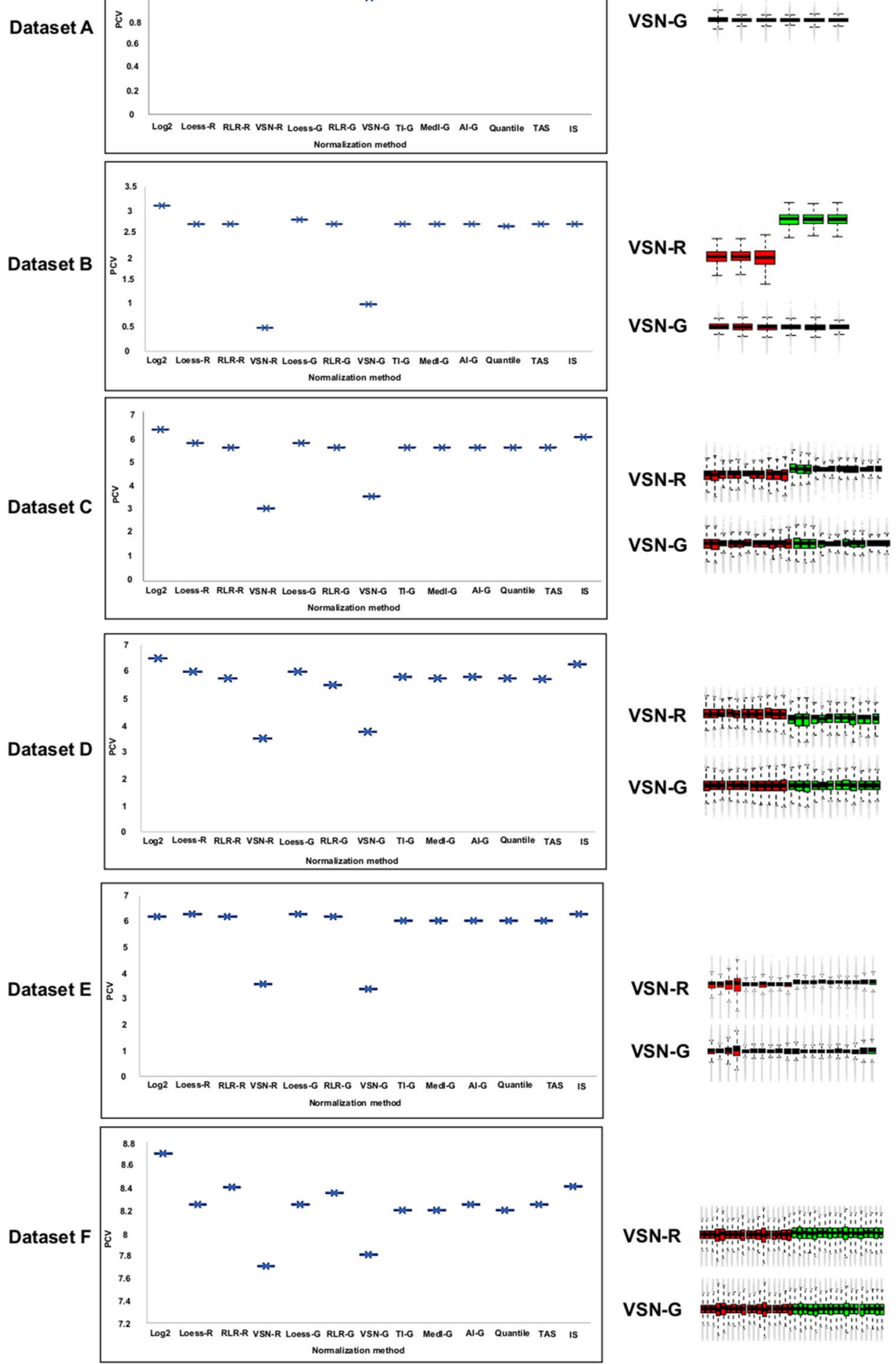

Fig. 3 I PCV plot: Quantitative assessment of twelve normalization methods indicates that VSN-G has less PCV in all datasets along with VSN-R in datasets B-F. II RLE plot: Qualitative analysis of methods with less PCV, by RLE plot revealed good inter group alignment only in VSN-G in all datasets 
as the optimal normalization method using 'Normalyzer' based evaluation.

\begin{abstract}
Assessment of VSN-G normalized data by cluster analysis Differentiators identified from data normalized by VSN-G method based on p-value, fold change and a combination of both were subjected to cluster analysis. Differentiators identified by all three criteria could segregate the comparison groups appropriately in datasets $\mathrm{A}, \mathrm{B}$ and D but not in dataset C, E and F (Fig. 4). Though VSN-G was identified as optimal normalization method based on PCV and RLE plots, the differentiators identified did not show consistent efficiency in clustering. In order to understand the contribution of VSN-G normalization to improper clustering of datasets $\mathrm{C}, \mathrm{E}$ and $\mathrm{F}$, differentiators identified by all three criteria, from data normalized with the remaining eleven methods were assessed for their clustering ability. The aim was to detect if any other normalization method could improve segregation of datasets $\mathrm{C}, \mathrm{E}$ and $\mathrm{F}$ while retaining the efficient segregation of datasets A, B and D in VSN-G normalized data.
\end{abstract}

\section{Assessment of data normalized by methods other than VSN-G by cluster analysis}

As observed in VSN-G normalized data, clusters obtained from data normalized with the remaining eleven methods yielded improper clustering in datasets $\mathrm{E}$ and $\mathrm{F}$. Thus the improper features of clusters i.e. formation of separate cluster by a few normal samples in datasets $\mathrm{E}$ and $\mathrm{F}$; segregation of a pair of normal samples (N9 and N18) with tumor samples in dataset F was taken as a consistent feature across normalized data for these two datasets and was not applied to eliminate a cluster as imprecise. While retaining these features, clear segregation of the remaining control and test samples was considered as acceptable clustering efficiency of datasets $\mathrm{E}$ and F. Based on this relaxed criteria, it is seen in Fig. 5 (Detailed dendrograms for cluster analysis is given in Additional file 1) and Table 2 that differentiators identified based on p-value efficiently segregate the comparison groups for data normalized by majority of methods. On the other hand, differentiators identified based on fold change could not segregate the comparison groups in majority of the datasets. The ability of differentiators obtained from the combination of p-value and foldchange to segregate sets therefore could be attributed to the influence of $\mathrm{p}$-value. Based on the above experimental evidence $\mathrm{p}$-value is chosen as the criteria for differentiator identification in this study.

Of the 11 normalization methods assessed, differentiators identified based on $\mathrm{p}$-value from data normalized by 3 methods (Loess-R, TI-G and AI-G) segregated the comparison groups precisely in all datasets (Fig. 5).
These were further evaluated using more stringent criteria to identify the most optimal method for biomarker discovery. The criteria was to sub-cluster the technical replicates, of control and test groups, belonging to each biological replicate precisely in datasets C, D and F. Dataset $\mathrm{E}$ was not subclustered as each sample was run only once [15]. A scoring system was used to achieve this, wherein the ability to segregate control and test groups was given a score of 2 . In dataset F, for every control which segregated separately from the major control cluster, a negative score of 1 was given. Thereafter for every correct subgrouping of the technical replicates of control and test, a score of 1 was given. The total score was calculated as score for precise clustering (2) + score of -1 for each control which clustered separately from the major control cluster in dataset F (not applicable to other datasets) + score for co-segregation of technical replicates in test and control (1)(Fig. 6).

As mentioned earlier, the efficiency of biomarkers lies in their ability to accurately stratify the heterogenous groups in a given population. It is evident from Fig. 6 that differentiators obtained from Loess- $\mathrm{R}$ normalized data could not only stratify the comparison groups precisely, but also had maximum sub-stratification score in the three large datasets assessed, thereby indicating its suitability for biomarker discovery.

\section{Discussion}

This study has addressed two previously unattended issues in analysis of quantitative SWATH-MS data, especially relevant to clinical proteomics-(i) experimental demonstration of ideal method of data normalization which does not diminish the vital features of the data necessary for segregation of comparison groups (ii) experimental verification of criteria for identification of differentiators. Carefully chosen sets of samples, mimicking the biological and experimental variations which can influence the data were included in the study. The reference set from public domain (dataset A) represented a quantitatively defined set wherein the differences in relative proportions of the constituents between samples made fold-differences in protein quantities predictable. The study set (datasets B-D) on the other hand represented the heterogeneity inherent to biological samples as in $S$ cells and those contributed by extraneous manipulations such as treatment with imatinib in S I IM and $R$ cells. The validation set (dataset $\mathrm{E}$ and $\mathrm{F}$ ) were analyzed to confirm the findings obtained in the earlier sets. While analysis of single sets in dataset A and B allowed to evaluate differences between quantitatively defined (dataset A) and undefined set (dataset B), multiple sets in dataset $\mathrm{C}, \mathrm{D}, \mathrm{E}$ and $\mathrm{F}$ allowed for evaluation of the consistency of observations within quantitatively undefined 


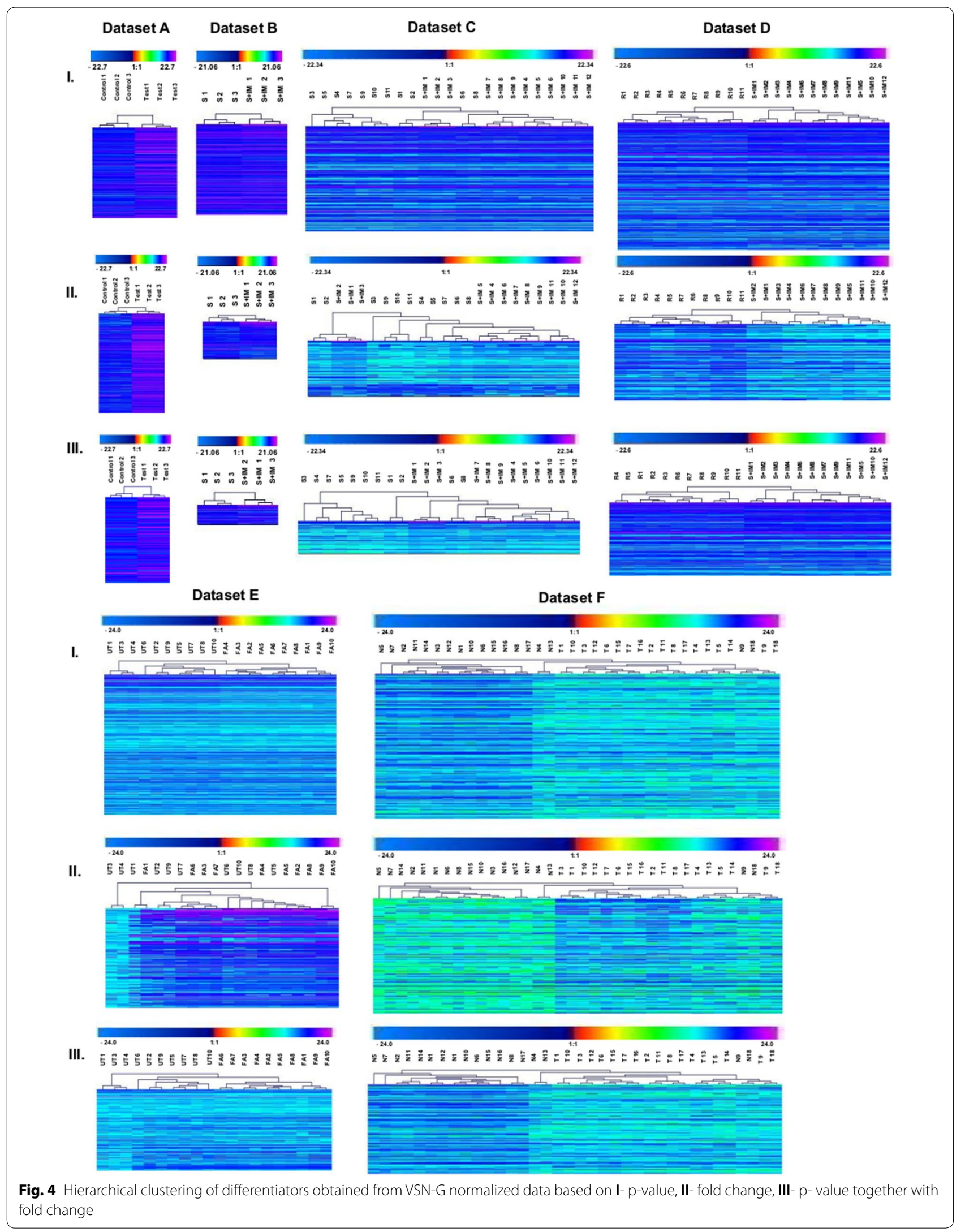




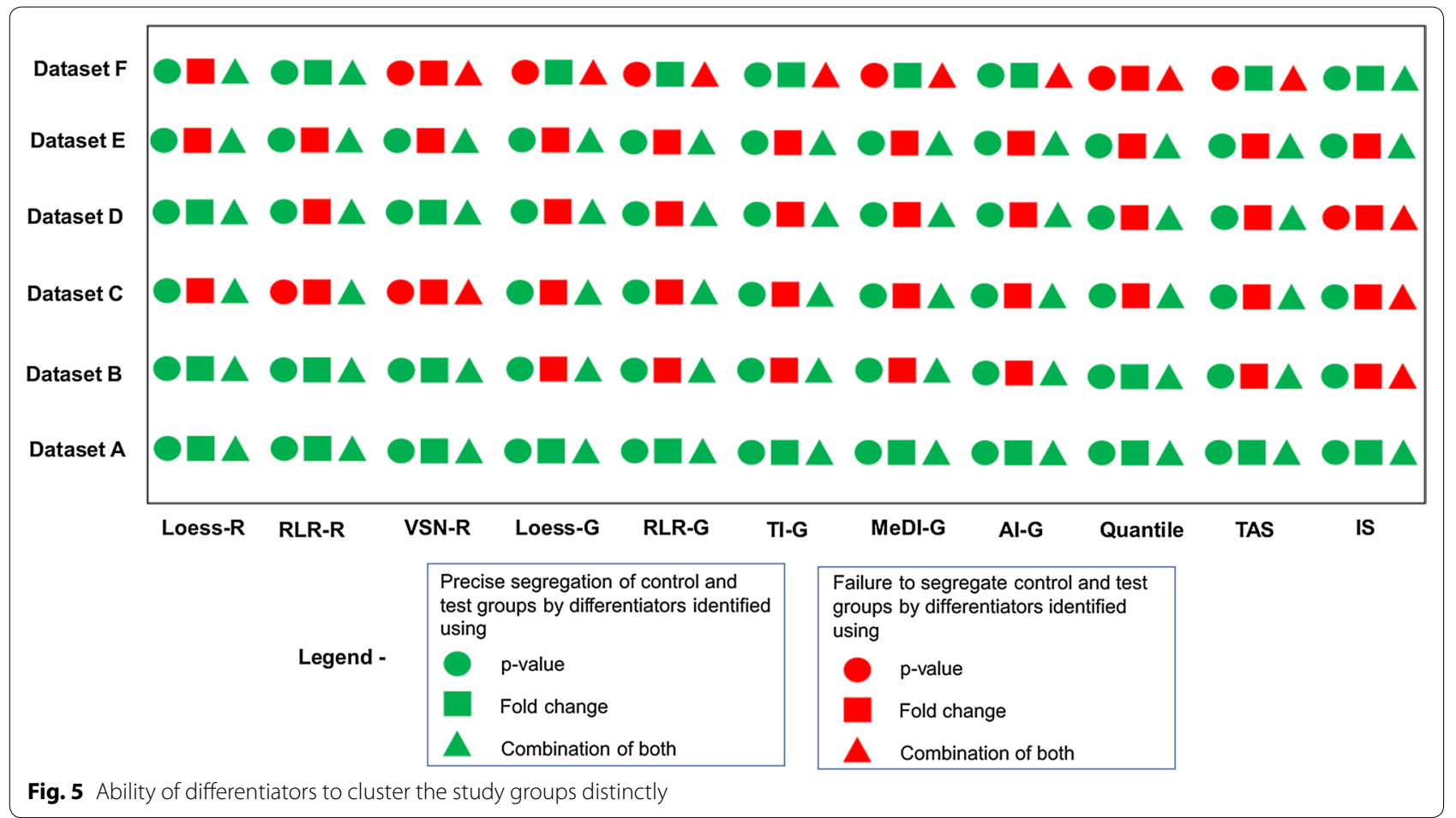

Table 2 Clustering efficiency of differentiators identified based on p-value, fold change and combination of both, from data normalized by 12 methods

\begin{tabular}{|c|c|c|c|}
\hline \multirow[t]{2}{*}{ Datasets } & \multicolumn{3}{|l|}{ Clustering efficiency } \\
\hline & p-value & Fold change & Both \\
\hline A & $100 \%$ (12/12 methods) & $100 \%$ (12/12 methods) & $100 \%$ (12/12 methods) \\
\hline B & $100 \%$ (12/12 methods) & $42 \%$ (5/12 methods) & $92 \%$ (11/12 methods) \\
\hline C & $75 \%$ (9/12 methods) & $0 \%$ (0/12 methods) & $75 \%$ (9/12 methods) \\
\hline $\mathrm{D}$ & $92 \%$ (11/12 methods) & $25 \%$ (3/12 methods) & $92 \%$ (11/12 methods) \\
\hline $\mathrm{E}$ & 100\% (12/12 methods) & $0 \%$ (0/12 methods) & $100 \%$ (12/12 methods) \\
\hline $\mathrm{F}$ & $42 \%$ (5/12 methods) & $66.6 \%$ (8/12 methods) & 25\% (3/12 methods) \\
\hline
\end{tabular}

sets. Further the reference, study and validation sets differed in the depth of spectral ion library, the choice of calibrants for retention time calibration as well as peak intensities. They are the prototypes of variation of methodologies observed in the reported literature. Analysis of these samples was designed to identify a strategy for normalization of SWATH-MS data which is applicable universally.

The differences in datasets throw light on certain valuable aspects of experimental design. Detection of greater number of proteins in dataset $\mathrm{A}, \mathrm{E}$ and $\mathrm{F}$ as compared to $\mathrm{B}, \mathrm{C}$ and $\mathrm{D}$ could be attributed to deeper spectral ion library. For dataset A, library was generated by pooling individual libraries of constituent human, yeast and $E$. coli peptides while for datasets $\mathrm{E}$ and $\mathrm{F}$ extensive human protein library was used. It could also be due to use of iRT peptides for retention time calibration in dataset $A$, $\mathrm{E}$ and $\mathrm{F}$ which allows high-quality spectral library generation. Retention time calibration of datasets B, C and D had been carried out using spiked peptides of $E$. coli beta galactosidase which span a limited range of retention times. This represents the studies where retention time calibration has been carried out using highly conserved and abundant endogenous peptides or spike-in peptides other than iRT $[19,20]$.

The extent of variation among un-normalized datasets, when assessed by RLE plot, showed a progressive increase from dataset A to D and F (Fig. 2). This increase 


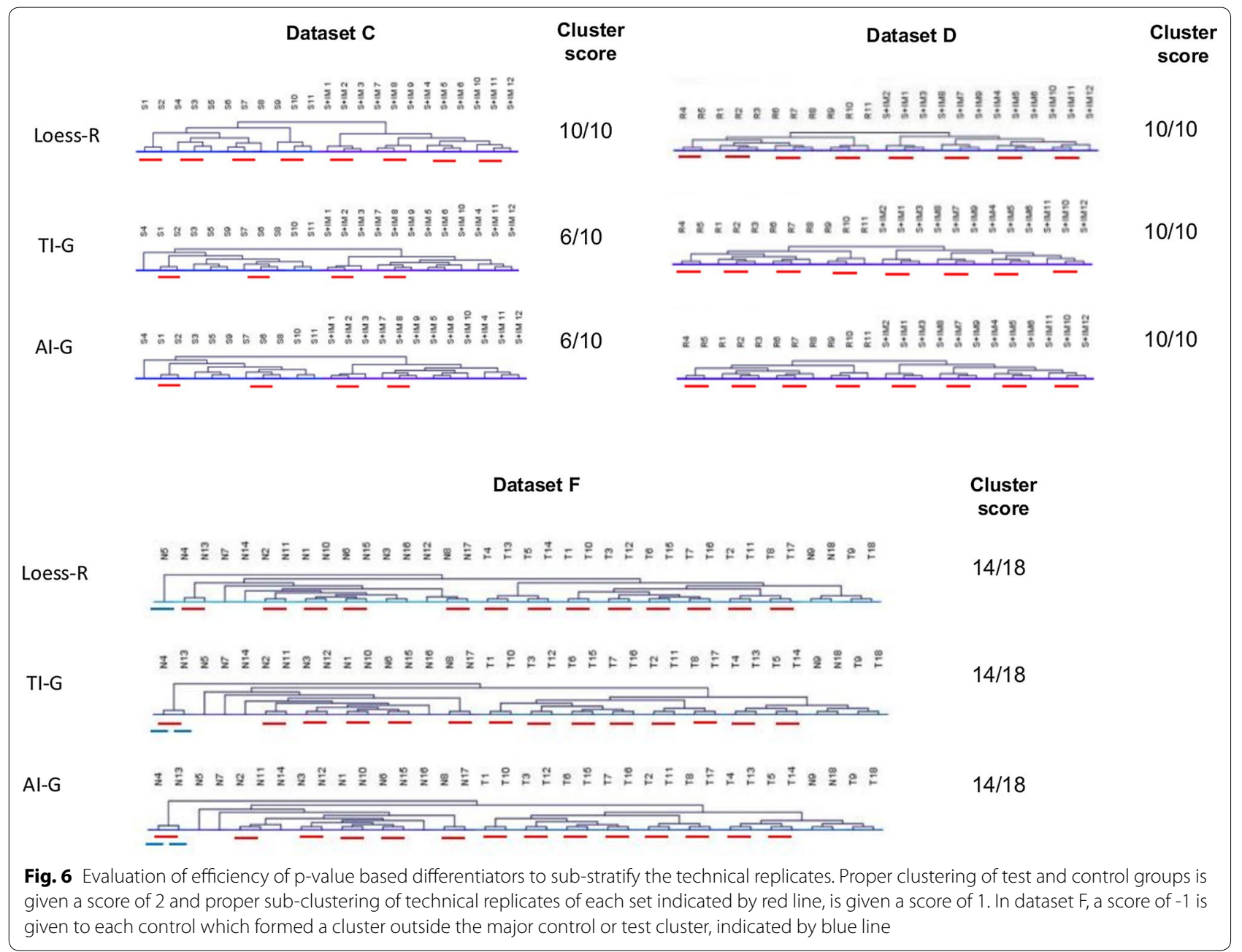

in variation could be attributed to increase in sample heterogeneity, as dataset $\mathrm{A}$, generated by addition of defined proportions of constituents, was less heterogenous and had more precise quantification. Dataset B on the other hand, was not a defined set and thus would exhibit variations inherent to any biological system. In datasets $C$ and $D$, the probability of variation increased as the heterogeneity increased due to inclusion of greater number of samples. Dataset E which also involved cell lines as in datasets $C$ and $D$, showed least variation which reflects precision in experimentation but is not commonly observed due to experimental errors. Dataset F included human samples which are inherently heterogenous. Such variations are a commonplace in clinical samples and reflect in the assessment of un-normalized data based on RLE plot. These observations emphasized the need for normalization of SWATH-MS data.

In most of the previously reported SWATH-MS studies, data has been normalized by TAS [21-29], median $[15,30-32]$, TI [33-36], quantile [37-39] and IS [40, 41] methods. In this study, to identify the optimum normalization method, datasets A-F were normalized using 10 normalization methods from Normalyzer and 2 methods from Marker View software, which included the above mentioned methods used in previous studies. Their normalization efficiency in Normalyzer was evaluated based on PCV and RLE plots. VSN-G was found to efficiently normalize not only dataset $\mathrm{A}$ with minimum variation but also datasets B-F with considerable variation (Fig. 3). This indicates that VSN-G could have a broad applicability for normalization of SWATH-MS data. VSN normalization has also been reported to efficiently normalize data generated by DIA using LTQ orbitrap [8]. Considering wide use of SWATH-MS for biomarker identification, the utility of VSN-G normalized data for biomarker discovery was assessed based on its ability to yield differentiators which segregate the comparison groups precisely.

Differentiators could be identified by comparing quantities of proteins in comparison groups based on $p$ value, fold change or combination of both. PubMed search 


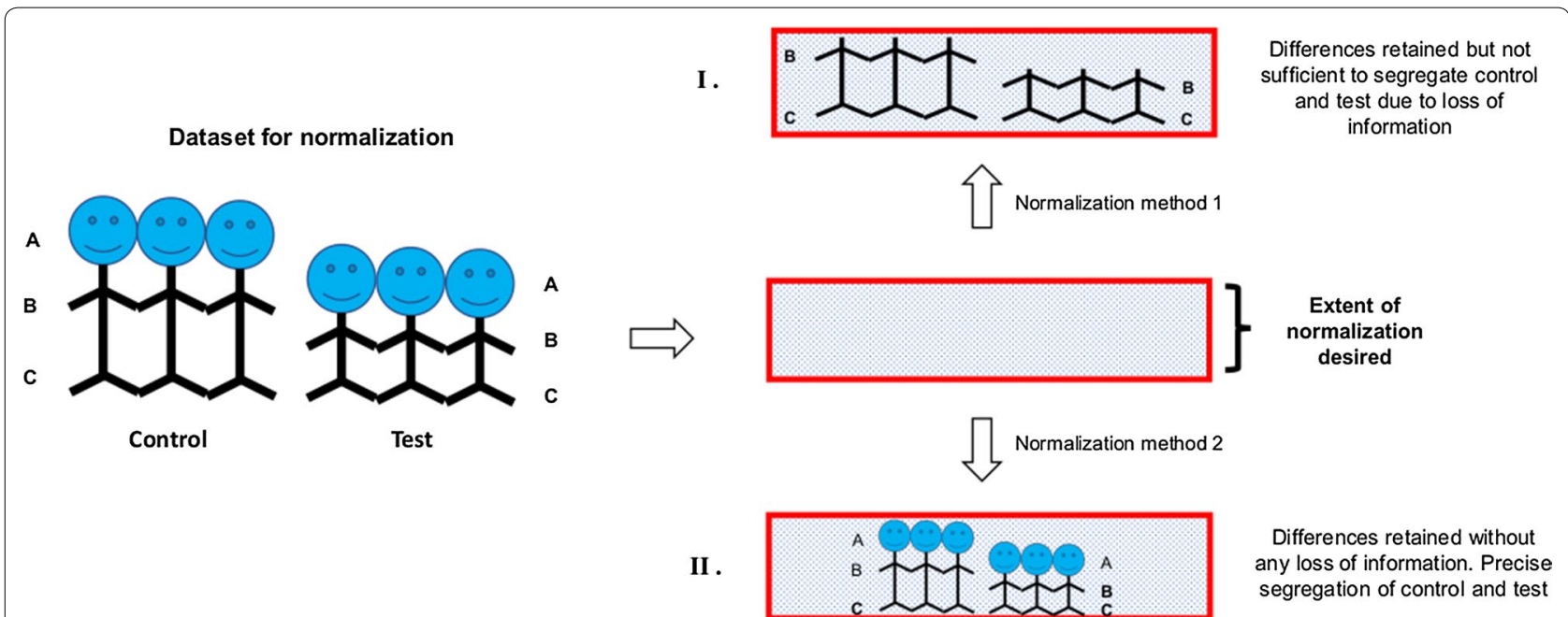

Fig. 7 Should application dictate the choice of normalization method for SWATH-MS data?

revealed that in over 134 human SWATH related publications in a span of 5 years (2012-Mar 2018), 45 studies (33.6\%) were aimed at identifying differentiators between control and test groups. Out of these 45 studies, we observed that 14 studies (31.1\%) used statistical significance (p-value), 6 studies (13.3\%) used fold change and 25 studies $(55.6 \%)$ used both ( $\mathrm{p}$ value and fold change) as criteria to identify differentiators. While experimental evidence for either choosing the criteria for differentiator identification ( $p$-value, fold change or combination of both) $[42,43]$ or their cut-off values [44] is available for transcriptomic data, no such studies are reported for MS data. Our study provides the first experimental evaluation of choice of criteria to identify differentiators from SWATH-MS data for biomarker discovery based on their ability to segregate comparison groups- an essential feature of biomarkers, by cluster analysis. Cluster analysis revealed that differentiators identified based on p-value, from data normalized by 12 methods, could segregate the comparison groups with maximum efficiency in 5 out of 6 datasets (Fig. 5). Hence p-value was chosen as the criteria for differentiator identification in this study.

VSN-G, though was identified as optimal normalization method based on PCV and RLE plots, the differentiators identified did not efficiently cluster the comparison groups in all datasets (Fig. 4), thereby raising question on its suitability for biomarker discovery. Loess-R, a method ranked lower in 'Normalyzer' based evaluation, on the other hand, yielded differentiators with maximum clustering as well as sub-clustering efficiency in all datasets assessed (Fig. 6), thereby making it suitable for biomarker discovery by SWATH-MS. This may be due to the differences in the assumptions made for normalization by these methods. The perceived treatment of data is depicted in Fig. 7. VSN-G aims at making the sample variances non-dependent on their mean intensity thereby bringing the samples onto the same scale. This assumption remarkably reduces the intensity differences between samples so as to achieve optimum normalization. However, the reduction in intensity differences is not conducive to identification of differentiators and in turn segregation of comparison groups (Fig. 7I) Loess normalization on the other hand, probably retains the differences between intragroup protein intensities by assuming non-linear relationship between biases in the data and the magnitude of protein intensity-a feature essential for segregation of comparison groups (Fig. 7II).

We thus propose that apart from statistically recommended criteria for evaluation of methods for normalization, a biologically relevant criteria like precise stratification of data should be assessed before a normalization method is used for biomarker identification from SWATH-MS data.

\section{Conclusion}

This study for the first time has identified VSN-G as method for optimum normalization of SWATH-MS data based on statistical criteria. Acknowledging the extensive use of this technology for biomarker discovery this study has also identified the normalization strategies conducive to this application. In the process, p-value based identification of differentiators has been demonstrated to be most suitable for biomarker discovery from SWATHMS data. While VSN-G normalization was not found conducive to biomarker discovery in this study, Loess- $R$ normalization was observed to retain features of the data 
necessary to yield differentiators which could segregate the comparison groups efficiently. The probable effect of two normalization methods on the data which are responsible for these observations are depicted in Fig. 7. The study has thus demonstrated the need to base the choice of normalization method on the application of the data.

\section{Additional file}

Additional file 1. Dendrograms representing hierarchical clustering of control and test groups based on differentiators obtained by p-value, fold change or both in both datasets.

\section{Abbreviations}

LC-MS: liquid chromatography Mass spectrometry; SWATH: Sequential window acquisition of all theoretical fragment ion spectra; DIA: data independent acquisition; IDA: information/data dependent acquisition; IM: imatinib; K562 S: imatinib sensitive cells; K562 S + IM: sensitive cells treated with imatinib; K562 $\mathrm{R}$ : cells resistant to imatinib; DTT: dithiothreitol; IAA: iodoacetamide; FA: formic acid; $\beta$-gal: E. coli beta galatosidase; $A C N$ : acetonitrile; iRT: indexed retention time; FDR: false discovery rate; XIC: extracted ion chromatogram; R: local normalization; G: global normalization; RLR: robust linear regression; VSN: variance stabilizing normalization; TI: total intensity; Al: average intensity; Medl-G: median intensity; TAS: total area sums; IS: internal standard; PCV: pooled intragroup coefficient of variation; RLE: relative log expression.

\section{Acknowledgements}

We acknowledge the support of mass spectrometry facility, ACTREC, Navi Mumbai.

\section{Authors' contributions}

MN has carried out experimental work, data analysis and initial writing of manuscript and has incorporated subsequent changes suggested by co-authors, AC has carried out data processing on 'Normalyzer'. AB and SK have carried out statistical analysis and RG has conceptualized the project and executed the same. All authors read and approved the final manuscript.

\section{Funding}

This work was funded by Intramural Research Grant of Tata Memorial Centre.

\section{Availability of data and materials}

The proteomics data generated for the study set in the current study have been deposited to the Proteome Xchange Consortium via the PRIDE [45] partner repository with the dataset identifier PXD009686. The reference dataset deposited by Navarro et al. [12], used for analysis in this study, has been obtained from Proteome Xchange Consortium (Identifier-PXD002952).

\section{Ethics approval and consent to participate}

Not applicable.

\section{Consent for publication}

Not applicable.

\section{Competing interests}

The authors declare that they have no competing interests.

\footnotetext{
Author details

${ }^{1}$ Advanced Centre for Treatment, Research and Education in Cancer, Tata Memorial Centre, Kharghar, Navi Mumbai 410210, India. ${ }^{2}$ BARC Training School Complex, Homi Bhabha National Institute, Anushakti Nagar, Mumbai 400094, India. ${ }^{3}$ Department of Plant Breeding, Swedish University of Agricultural Sciences, Alnarp, Sweden. ${ }^{4}$ Section of Biostatistics, Centre for Cancer Epidemiology, Tata Memorial Centre, Kharghar, Navi Mumbai 410210, India.
}

Received: 28 March 2019 Accepted: 24 May 2019

Published online: 31 May 2019

\section{References}

1. Diamandis EP. Mass spectrometry as a diagnostic and a cancer biomarker discovery tool opportunities and potential limitations. Mol Cell Proteomics. 2004;3(4):367-78.

2. Luo Y, MokTS, Lin X, Zhang W, Cui Y, Guo J, Chen X, Zhang T, Wang T. SWATH-based proteomics identified carbonic anhydrase 2 as a potential diagnosis biomarker for nasopharyngeal carcinoma. Sci Rep. 2017:7:41191.

3. Ralhan R, DeSouza LV, Matta A, Tripathi SC, Ghanny S, Gupta SD, Bahadur S, Siu KM. Discovery and verification of head-and-neck cancer biomarkers by differential protein expression analysis using iTRAQ labeling, multidimensional liquid chromatography, and tandem mass spectrometry. Mol Cell Proteomics. 2008;7(6):1162-73.

4. Gillet LC, Navarro P, Tate S, Röst H, Selevsek N, Reiter L, Bonner R, Aebersold R. Targeted data extraction of the MS/MS spectra generated by data-independent acquisition: a new concept for consistent and accurate proteome analysis. Mol Cell Proteomics. 2012;11(6):0111-016717.

5. Domon B, Aebersold R. Options and considerations when selecting a quantitative proteomics strategy. Nat Biotechnol. 2010;28(7):710-21.

6. Karpievitch YV, Dabney AR, Smith RD. Normalization and missing value imputation for label-free LC-MS analysis. BMC Bioinform. 2012;13(16):S5.

7. Callister SJ, Barry RC, Adkins JN, Johnson ET, Qian W-J, Webb-Robertson B-JM, Smith RD, Lipton MS. Normalization approaches for removing systematic biases associated with mass spectrometry and label-free proteomics. J Proteome Res. 2006;5(2):277-86.

8. Valikangas T, Suomi T, Elo LL. A systematic evaluation of normalization methods in quantitative label-free proteomics. Brief Bioinform. 2018:19(1):1-11.

9. Kultima K, Nilsson A, Scholz B, Rossbach UL, Fälth M, Andrén PE. Development and evaluation of normalization methods for label-free relative quantification of endogenous peptides. Mol Cell Proteomics. 2009;8(10):2285-95.

10. Chawade A, Alexandersson E, Levander F. Normalyzer: a tool for rapid evaluation of normalization methods for omics data sets. J Proteome Res. 2014;13(6):3114-20.

11. Fu J, Tang J, Wang Y, Cui X, Yang Q, Hong J, Li X, Li S, Chen Y, Xue W, et al. Discovery of the consistently well-performed analysis chain for SWATH-MS based pharmacoproteomic quantification. Front Pharmacol. 2018;9:681.

12. Navarro P, Kuharev J, Gillet LC, Bernhardt OM, MacLean B, Rost HL, Tate SA, Tsou CC, Reiter L, Distler U, et al. A multicenter study benchmarks software tools for label-free proteome quantification. Nat Biotechnol. 2016;34(11):1130-6.

13. Xiong $L$, Zhang J, Yuan B, Dong $X$, Jiang $X$, Wang Y. Global proteome quantification for discovering imatinib-induced perturbation of multiple biological pathways in K562 human chronic myeloid leukemia cells. J Proteome Res. 2010;9(11):6007-15.

14. Arvaniti K, Papadioti A, Kinigopoulou M, Theodorou V, Skobridis K Tsiotis G. Proteome changes induced by imatinib and novel imatinib derivatives in $\mathrm{K} 562$ human chronic myeloid leukemia cells. Proteomes. 2014;2(3):363-81.

15. Tan SLW, Chadha S, Liu Y, Gabasova E, Perera D, Ahmed K, Constantinou S, Renaudin X, Lee M, Aebersold R, et al. A class of environmental and endogenous toxins induces BRCA2 haploinsufficiency and genome instability. Cell. 2017;169(6):1105-18.

16. Guo T, Kouvonen P, Koh CC, Gillet LC, Wolski WE, Rost HL. Rapid mass spectrometric conversion of tissue biopsy samples into permanent quantitative digital proteome maps. Nat Med. 2015;21(4):407-13.

17. Bradford MM. A rapid and sensitive method for the quantitation of microgram quantities of protein utilizing the principle of protein-dye binding Anal Biochem. 1976;72:248-54.

18. Rosenberger G, Koh CC, Guo T, Rost HL, Kouvonen P, Collins BC, Heusel M, Liu Y, Caron E, Vichalkovski A, et al. A repository of assays to quantify 10,000 human proteins by SWATH-MS. Sci Data. 2014;1:140031.

19. Parker SJ, Rost H, Rosenberger G, Collins BC, Malmstrom L, Amodei D, Venkatraman V, Raedschelders K, Van Eyk JE, Aebersold R. Identification 
of a set of conserved eukaryotic internal retention time standards for data-independent acquisition mass spectrometry. Mol Cell Proteomics. 2015;14(10):2800-13.

20. Hou G, Lou X, Sun Y, Xu S, Zi J, Wang Q, Zhou B, Han B, Wu L, Zhao X, et al. Biomarker discovery and verification of esophageal squamous cell carcinoma using integration of SWATH/MRM. J Proteome Res. 2015;14(9):3793-803.

21. Ortea I, Rodriguez-Ariza A, Chicano-Galvez E, Arenas Vacas MS, Jurado Gamez B. Discovery of potential protein biomarkers of lung adenocarcinoma in bronchoalveolar lavage fluid by SWATH MS data-independent acquisition and targeted data extraction. J Proteomics. 2016;138:106-14.

22. Chaudhari PR, Charles SE, D'Souza ZC, Vaidya MM. Hemidesmosomal linker proteins regulate cell motility, invasion and tumorigenicity in oral squamous cell carcinoma derived cells. Exp Cell Res. 2017;360(2):125-37.

23. Arya S, Emri E, Synowsky SA, Shirran SL, Barzegar-Befroei N, Peto T, Botting $\mathrm{CH}$, Lengyel I, Stewart AJ. Quantitative analysis of hydroxyapatite-binding plasma proteins in genotyped individuals with late-stage age-related macular degeneration. Exp Eye Res. 2018:172:21-9.

24. Batkulwar K, Godbole R, Banarjee R, Kassaar O, Williams RJ, Kulkarni MJ. Advanced glycation end products modulate amyloidogenic APP processing and tau phosphorylation: a mechanistic link between glycation and the development of Alzheimer's disease. 2018;9(5):988-1000

25. Schillaci O, Fontana S. Exosomes from metastatic cancer cells transfer amoeboid phenotype to non-metastatic cells and increase endothelial permeability: their emerging role in tumor heterogeneity. Sci Rep. 2017;7(1):4711.

26. Lewandowska AE, Macur K, Czaplewska P, Liss J, Lukaszuk K, Oldziej S. Qualitative and quantitative analysis of proteome and peptidome of human follicular fluid using multiple samples from single donor with LCMS and SWATH methodology. J Proteome Res. 2017;16(8):3053-67.

27. Lin Q, Lim HS, Lin HL, Tan HT, Lim TK, Cheong WK, Cheah PY, Tang CL, Chow PK, Chung MC. Analysis of colorectal cancer glyco-secretome identifies laminin beta-1 (LAMB1) as a potential serological biomarker for colorectal cancer. Proteomics. 2015;15(22):3905-20.

28. Korwar AM, Vannuruswamy $G$, Jagadeeshaprasad MG, Jayaramaiah RH, Bhat S, Regin BS, Ramaswamy S, Giri AP, Mohan V, Balasubramanyam M, et al. Development of diagnostic fragment ion library for glycated peptides of human serum albumin: targeted quantification in prediabetic, diabetic, and microalbuminuria plasma by parallel reaction monitoring, SWATH, and MSE. Mol Cell Proteomics. 2015;14(8):2150-9.

29. Tan HT, Lim TK, Richards AM, Kofidis T, Teoh KL, Ling LH, Chung MC. Unravelling the proteome of degenerative human mitral valves. Proteomics. 2015;15(17):2934-44.

30. Netherton JK, Hetherington L, Ogle RA, Velkov T, Baker MA. Proteomic analysis of good- and poor-quality human sperm demonstrates that several proteins are routinely aberrantly regulated. Biol Reprod. 2018;99(2):395-408.

31. Nemeth J, Vongrad V, Metzner KJ, Strouvelle VP, Weber R, Pedrioli P, Aebersold R, Gunthard HF, Collins BC. In vivo and in vitro proteome analysis of human immunodeficiency virus (HIV)-1-infected, human CD4+T cells. Mol Cell Proteomics. 2017;16(4 suppl 1):S108-23.

32. Chang RY, Etheridge N, Nouwens AS, Dodd PR. SWATH analysis of the synaptic proteome in Alzheimer's disease. Neurochem Int. 2015:87:1-12.

33. Blattmann $P$, Henriques D, Zimmermann M, Frommelt F, Sauer U, Saez-Rodriguez J, Aebersold R. Systems pharmacology dissection of cholesterol regulation reveals determinants of large pharmacodynamic variability between cell lines. Cell systems. 2017;5(6):604-19.

34. Zhang X, Walsh T, Atherton JJ, Kostner K, Schulz B, Punyadeera C. Identification and validation of a salivary protein panel to detect heart failure early. Theranostics. 2017;7(18):4350-8.

35. Bjelosevic S, Pascovici D, Ping H, Karlaftis V, Zaw T, Song X, Molloy MP, Monagle P, Ignjatovic V. Quantitative age-specific variability of plasma proteins in healthy neonates, children and adults. Mol Cell Proteomics. 2017:16(5):924-35.

36. Liu Y, Chen J, Sethi A, Li QK, Chen L, Collins B, Gillet LC, Wollscheid B, Zhang H, Aebersold R. Glycoproteomic analysis of prostate cancer tissues by SWATH mass spectrometry discovers $\mathrm{N}$-acylethanolamine acid amidase and protein tyrosine kinase 7 as signatures for tumor aggressiveness. Mol Cell Proteomics. 2014;13(7):1753-68.

37. Liu Y, Borel C, Li L, Muller T, Williams EG, Germain PL, Buljan M, Sajic T, Boersema PJ, Shao W, et al. Systematic proteome and proteostasis profiing in human Trisomy 21 fibroblast cells. Nat Commun. 2017;8(1):1212.

38. Chen L, Li J, Guo T, Ghosh S, Koh SK, Tian D, Zhang L, Jia D, Beuerman RW, Aebersold R, et al. Global metabonomic and proteomic analysis of human conjunctival epithelial cells (IOBA-NHC) in response to hyperosmotic stress. J Proteome Res. 2015;14(9):3982-95.

39. Orellana CA, Marcellin E, Schulz BL, Nouwens AS, Gray PP, Nielsen LK. High-antibody-producing Chinese hamster ovary cells up-regulate intracellular protein transport and glutathione synthesis. J Proteome Res. 2015;14(2):609-18.

40. Martins-Marques T, Anjo SI, Pereira P, Manadas B, Girao H. Interacting network of the gap junction (GJ) Protein Connexin43 (Cx43) is modulated by ischemia and reperfusion in the heart. Mol Cell Proteomics. 2015;14(11):3040-55.

41. Villeneuve LM, Stauch KL, Fox HS. Proteomic analysis of the mitochondria from embryonic and postnatal rat brains reveals response to developmental changes in energy demands. J Proteomics. 2014;109:228-39.

42. McCarthy DJ, Smyth GK. Testing significance relative to a fold-change threshold is a TREAT. Bioinformatics. 2009;25(6):765-71.

43. Xiao Y, Hsiao T-H, Suresh U, Chen H-IH, Wu X, Wolf SE, Chen YA. Novel significance score for gene selection and ranking. Bioinformatics. 2014;30(6):801-7.

44. Dalman MR, Deeter A, Nimishakavi G, Duan Z-H. Fold change and p-value cutoffs significantly alter microarray interpretations. BMC Bioinformatics. 2012;13(2):S11.

45. Vizcaíno JA, Csordas A, del Toro N, Dianes JA, Griss J, Lavidas I, Mayer G, Perez-Riverol Y, Reisinger F, Ternent T, et al. 2016 update of the PRIDE database and its related tools. Nucleic acids Res. 2016;44(1):447-56.

46. Loke MF, Ng CG, Vilashni Y, Lim J, Ho B. Understanding the dimorphic lifestyles of human gastric pathogen Helicobacter pylori using the SWATH-based proteomics approach. Sci Rep. 2016;6:26784.

47. Lewandowska AE, Macur K, Czaplewska P, Liss J, Lukaszuk K, Oldziej S. Human follicular fluid proteomic and peptidomic composition quantitative studies by SWATH-MS methodology. Applicability of high pH RPHPLC fractionation. J Proteomics. 2018;191:131-42.

\section{Publisher's Note}

Springer Nature remains neutral with regard to jurisdictional claims in published maps and institutional affiliations. 